\title{
INTESTINAL GAS CONTENT AND DISTRIBUTION IN HEALTH AND IN PATIENTS WITH FUNCTIONAL GUT SYMPTOMS
}

Short title: abdominal gas

Alvaro Bendezú, M.D ${ }^{1}$, Elizabeth Barba, M.D. ${ }^{1}$, Emanuel Burri, M.D. ${ }^{1,2}$, Daniel Cisternas ${ }^{1,3}$, Carolina Malagelada, M.D. ${ }^{1}$, Santi Segui, M.D. ${ }^{4}$, Anna Accarino, M.D. ${ }^{1}$, Sergi Quiroga, M.D. ${ }^{5}$, Eva Monclus ${ }^{6}$, Isabel Navazo, M.D. ${ }^{6}$, Juan-R. Malagelada, M.D. ${ }^{1}$, and Fernando Azpiroz, M.D. ${ }^{1}$

1. Digestive System Research Unit, University Hospital Vall d'Hebron; Centro de Investigación Biomédica en Red de Enfermedades Hepáticas y Digestivas (Ciberehd), Barcelona, Spain; Departament de Medicina, Universitat Autònoma de Barcelona, 08193 Bellaterra (Cerdanyola del Vallès), Spain.

2. Current address: Department of Internal Medicine, Stadspital Triemli, Zürich, Switzerland.

3. Current address: Departamento de Gastroenterología. Facultad de Medicina. Pontificia Universidad Católica de Chile, Santiago, Chile.

4. Computer Vision Center, Bellaterra, Spain

5. Radiology Department, University Hospital Vall d'Hebron, Barcelona, Spain

6. Departamento de Lenguajes y Sistemas Informáticos, Universitat Politécnica de Catalunya, Spain.

Abbreviations: EMG: electromyography

Corresponding author: $\quad$ Fernando Azpiroz, M.D.

Digestive System Research Unit

Hospital General Vall d'Hebron

08035-Barcelona, Spain

Phone: (34) 932746222

Fax: (34) 934894456

E-mail: azpiroz.fernando@gmail.com 


\begin{abstract}
Background. The precise relation of intestinal gas to symptoms, particularly abdominal bloating and distension remains incompletely elucidated. Our aim was to define the normal values of intestinal gas volume and distribution and to identify abnormalities in relation to functional-type symptoms.

Methods. Abdominal CT scans were evaluated in healthy subjects $(n=37)$ and in patients in 3 conditions: basal (when they were feeling well; $n=88$ ), during an episode of abdominal distension $(n=82)$ and after a challenge diet $(n=24)$. Intestinal gas content and distribution were measured by an original analysis program. Identification of patients outside the normal range was performed by machine learning techniques (one-class classifier). Results are expressed as median [IQR] or mean $\pm S E$, as appropriate.
\end{abstract}

Key results. In healthy subjects the gut contained $95[71,141] \mathrm{mL}$ gas distributed along the entire lumen. No differences were detected between patients studied under asymptomatic basal conditions and healthy subjects. However, either during a spontaneous bloating episode or once challenged with a flatulogenic diet, luminal gas was found to be increased and/or abnormally distributed in about one fourth of the patients. These patients detected outside the normal range by the classifier exhibited a significantly greater number of abnormal features than those within the normal range $(3.7 \pm 0.4$ vs $0.4 \pm 0.1$; $p<0.001)$.

Conclusions \& Inferences. The analysis of a large cohort of subjects using original techniques provides unique and heretofore unavailable information on the volume and distribution of intestinal gas in normal conditions and in relation to functional gastrointestinal symptoms. 
Key words: intestinal gas; abdominal distension; abdominal bloating; functional gut disorders. 


\section{Key messages}

- Our study, including a relatively large cohort of subjects and applying original analysis techniques, provides unique and heretofore unavailable information on the volume and distribution of intestinal gas in normal conditions and in relation to functional gastrointestinal symptoms.

- During asymptomatic intervals, patients who complain of gas-related symptoms did not show differences in intestinal gas content and distribution as compared to healthy subjects.

- During an episode of abdominal bloating, the same patients exhibited relatively minor abnormalities in intestinal gas, but using machine learning techniques a significant proportion of them were found outside the normal range. 


\section{INTRODUCTION}

The volume and distribution of gas within the gastrointestinal tract is tightly controlled by a complex homeostasis that involves gas production, mucosal absorption, consumption by microbiota, intraluminal propulsion and anal evacuation (1). Although patients and physicians frequently attribute symptoms, such as abdominal bloating, distension and flatulence, to accumulation of gas in the gut, the precise role of gas in this context has not been convincingly elucidated $(2 ; 3)$.

Experimental human studies have shown that, in response to jejunal gas infusion, patients with abdominal bloating develop gas retention and symptoms, whereas healthy subjects tolerate and rapidly expel the infused gas loads (4-9). In apparent conflict with these earlier observations, when such patients complaining of abdominal distension were specifically investigated, no major abnormalities in intraluminal gas volume and/or distribution were detected either during basal conditions or during symptomatic episodes with abdominal distension (10-14). However, due to the limited sample size of healthy subjects and patients included in previous individual studies, undetected abnormalities related to a type II error could not be excluded.

The goal of the present study was to define the normal values of intestinal gas volume and distribution, and to identify abnormalities in intestinal gas in relation to functional-type abdominal symptoms. To this aim, we pooled the data from previous studies in healthy subjects and in patients with symptoms commonly ascribed to excess intestinal gas, such as bloating, abdominal distension and flatulence, performed over the past 10 years in our 
laboratory under a standard protocol for abdominal CT imaging (10-14). In the present study, morphovolumetric analysis techniques previously developed in our laboratory have been applied together with new tools specifically developed under the current project. Furthermore, taking advantage of the relatively large pool of subjects, we applied machine learning techniques to identify patients out of the normal range as defined in healthy subjects.

\section{MATERIAL AND METHODS}

\section{Participants}

Groups of patients (see Experimental design below) complaining of bloating, abdominal distension, and flatulence were included in the study and compared to healthy individuals with no gastrointestinal complaints. All participants had filled-out a digestive symptoms questionnaire based on Rome criteria; this information was used to establish a diagnosis in patients and to verify the absence of symptoms in healthy subjects. The protocols of the different studies had been approved by the Institutional Review Board of the University Hospital Vall d'Hebron and all subjects gave their written informed consent to participate in the studies.

\section{CT scanning}

Abdominal CT scans were obtained with a helical multislice CT scanner, exposure $120 \mathrm{kV}$ and $50 \mathrm{mAs}$, using the available dose reduction options (tube current modulation). Section thickness and image reconstruction depended on the scanner model $(2.5-\mathrm{mm}$ thickness, $1.6-\mathrm{mm}$ interval reconstruction with Mx8000, Philips Medical Systems, Best, The Netherlands; 2-mm thickness, 1.5- 
$\mathrm{mm}$ interval reconstruction with Somatom Sensation 16 or Somatom Definition AS, Siemens Medical Solutions, Forchheim, Germany). Images were obtained in the supine position during a single breath-hold. No oral or intravenous contrast medium was administered. At the time of each CT scan, the participant's subjective sensation of abdominal distension was measured on a graphic rating scale graded from 0 (no distension) to 6 (extremely severe distension).

\section{Experimental design}

All participants underwent at least one abdominal CT scan. A group of patients with episodic abdominal distension, i.e. severe distension episodes in contrast to mild or no distension during basal conditions, were studied on two different symptoms status (paired studies): at baseline when they felt their abdomen showed minimal or no distension and during a spontaneous episode of severe distension. Another group of patients with the primary complain of flatulence, but also affected by bloating and other abdominal symptoms, was studied after a challenge diet; in them imaging was performed after a 3-day high- flatulogenic diet and 6-h after ingestion of a test meal. The diet included 3 daily portions of the following: a) bread, cereals and pastries made of whole wheat or corn; b) beans, soya bean, corn, broad bean, peas; c) Brussels' sprouts, cauliflower, broccoli, cabbage, celery, onion, leek, garlic, artichoke; and d) banana, fig, peach, grapes, prunes. The test meal consisted in cooked beans with sausage, whole wheat bread and canned peach. CT scanning and analyses were performed blindly, with the investigator unaware of the condition 
(patient or healthy subject) and the symptom status (basal or symptomatic) of the participants.

\section{Data analysis}

Morphovolumetric analysis of CT images was performed using an original software program specifically developed in our laboratory and previously described in detail $(11 ; 15)$. To measure the volume of gas within the gut, images were filtered with a user-defined threshold to separate gas from tissues. The program allows measurement of total gas volume within the abdominal cavity and of the segmental volumes corresponding to selected regions of the gut. For this study two new tools were developed for measuring the distribution of gas within the entire small bowel (bubble size and number) and in the region potentially corresponding to the terminal ileum (defined as an antero-posterior cylinder with a diameter equivalent to $1 / 4$ the distance between the anterior iliac spines and tangential to the ileocecal valve).

The software used in this study was built with two open-source toolkits: the Insight Segmentation and Registration toolkit (16) and the Visualization toolkit, both used for 3D computer graphics, image processing and visualization (17).

\section{Statistical analysis}

Statistical analysis was performed using the SPSS 18.0 statistical package. Mean \pm SE or median values and interquartile range $[\mathrm{IQR}]$ of the features measured were calculated in each group of subjects, as appropriate. The normal range for the different features measured was established by the 5- 
$95 \%$ of the values in healthy subjects. The Kolmogorov-Smirnov test was used to check the normality of data distribution. Parametric normally-distributed data were compared by Student's t-test for paired or unpaired data; otherwise, the Wilcoxon signed rank test was used for paired data and the Mann-Whitney $U$ test for unpaired data. Distribution of abnormalities between groups was evaluated by the chi-square test. The effect of age and BMI was analyzed by dividing the subjects (within subgroups and the whole group) in the two halves based on the values of these features and comparing the data of the upper versus the lower half. Differences were considered significant at a $p$ value $<$ 0.05

\section{Algorithm for identification of patients with abnormal intestinal gas distribution}

Each subject was defined by 8 features (dimensions) related to the volume of gas in the different gut compartments (Figures 1 and 2). Based on the data in healthy subjects, used as a training set, a program (one-class support vector machine) calculated the function that best defined the normal range allowing $5 \%$ outliers: based on the examples of the training set, the program draws the hyperplane that defines the normal range in a multidimensional space. In essence, the program instead of defining cut-offs for each individual feature, finds the combination of features that best characterizes the normal range and constructs the function that defines the boundary plane (18). Using this algorithm, the different subgroups of patients were then tested to identify those outside the normal range. 
Patients identified as abnormal were then analyzed by the K-means clustering technique (Lloyd's algorithm) to determine the existence of subgroups (19). This algorithm uses an iterative refinement technique: starting from randomly selected subgroups, each case is assigned to the subgroup with the nearest mean (centroid of the cluster), and the new mean is calculated; the procedure is repeated until convergence is reached.

Cross validation of the algorithm in the group of healthy subjects, initially used as the training set, was performed by partitioning the data sample into 2 subsets: one was used for training (training set) and the other was then tested (validation test set). Specifically, using the leave-one-out method (20), one subject from the original data sample was retained as the testing set, and the remainder (all but himself) were used as the training set; the procedure was repeated for all subjects to define the outliers.

\section{RESULTS}

\section{Healthy subjects}

In healthy subjects ( $\mathrm{n}=37 ; 27$ women, 10 men; age range 19-62 years) the abdomen contained a median of $95[71,141] \mathrm{mL}$ gas, which was distributed throughout the digestive tract from the stomach down to the pelvic colon, but overall the largest part $(69[58,83] \%)$ was located within the colon (Figures 1 and 2). In two healthy subjects the total volume of gas was well above the rest, owing to larger colonic gas content.

In 11 subjects imaging was performed during the postprandial period (less than $2 \mathrm{~h}$ after ingestion of a meal), and in them gas volume and distribution was not different than in another 26 subjects studied during fasting 
(more than 2-h after ingestion of the last meal). Hence, the whole set of healthy subjects was included as training set to develop a classifier, that defined the normal range based on the combination of all the features analyzed (total and segmental gas content). Cross-validation by the leave-one-out method identified 2 outliers that fell out of the normal range (Figure 3), which were the two subjects with excessive colonic gas content mentioned earlier.

\section{Patients}

All patients were diagnosed as having functional disorders based on Rome III criteria (21), 44 of them as functional bloating $(9.8 \pm 0.8$ bowel movement/wk, $4.1 \pm 0.2$ score on the Bristol stool form scale), 52 constipationpredominant IBS (1.5 \pm 0.2 bowel movements/wk, $2.3 \pm 0.3$ Bristol score); 17 with alternating IBS (9.0 \pm 2.6 bowel movements/wk, $3.4 \pm 0.3$ Bristol score), 4 with functional abdominal pain (7.0 \pm 0.0 bowel movements/wk, $4.1 \pm 0.3$ Bristol score) and 1 diarrhea-predominant IBS.

\section{Basal conditions}

Patients complaining of episodic abdominal distension studied during basal conditions ( $n=88$; 82 women, 6 men; age range 19-74 yrs; mean abdominal symptom score 2.0 \pm 0.2 ) exhibited a gas pattern (volume and distribution) similar as in healthy subjects (Figures 1 and 2). Overall, the number of individual features outside the normal range (defined as 5-95 percentile in healthy subjects) was not significantly different than in healthy subjects $(0.4 \pm 0.1$ vs $0.4 \pm 0.2 ; p=0.955$ ). The proportion of patients identified by the classifier 
outside the normal range was also similar to that of outliers identified by crossvalidation in healthy subjects (Figure 3).

\section{Spontaneous bloating episodes}

A subset of these patients with episodic abdominal distension $(n=82 ; 77$ women, 5 men; age range 19-74 yrs) were re-evaluated during an episode of perceived abdominal distension ( $4.7 \pm 0.1$ subjective distension score; $p<0.001$ vs basal) and the CT scan showed a modest, but significant, increase in abdominal gas as compared to their basal values. The increment was by-andlarge located in the colon (Figures 1 and 2). Overall, the number of individual features related to gas volume and distribution outside the normal range $(1.1 \pm 0.2)$ was significantly greater than during basal conditions $(p<0.001)$ by paired analysis) and than in the group of healthy subjects $(p=0.042)$. Using the classifier, a significantly greater proportion of patients during an episode of spontaneous distension fell outside the normal range (Figure 3).

\section{Challenge diet}

After the challenge diet, patients that complained of excessive flatulence and bloating ( $\mathrm{n}=24 ; 14$ women, 10 men; age range $35-73$ yrs) reported increased sensation of abdominal distension $(4.4 \pm 0.2$ score vs $3.4 \pm 0.3$ score before challenge; $p=0.001)$. The pattern of intestinal gas on the challenge diet resembled that observed in patients during a spontaneous episode of abdominal distension, except for a slightly larger accumulation of gas in the pelvic colon (Figures 1 and 2). The number of individual features outside the normal range $(1.1 \pm 0.3)$, as well as the proportion of patients detected as 
abnormal by the classifier on the challenge diet was not significantly different than in patients during spontaneous distension (Figure 3).

\section{Gas distribution within the small bowel}

A further in depth analysis was performed by measuring intraluminal gas distribution in the small bowel. In healthy subjects the majority of gas within the small bowel was dispersed in bubbles of a wide size range (median volume of $0.08[0.06,0.09] \mathrm{mL})$ and a small proportion of that gas $(15.1[4.5,24.8] \%)$ was located in an area approximately corresponding to the terminal ileum. The spectrum of gas distribution, in terms of bubble size and number of gas bubbles either in the entire small bowel or in the region of the terminal ileum did not show consistent differences among healthy subjects and the 3 clinical conditions in patients (baseline, distension episodes and challenge diet) (Figure 4).

\section{Characterization of patients identified out of the normal range.}

Patients identified by the classifier outside the normal range, either during episodes of spontaneous distension or after the challenge diet, exhibited a large number of abnormal features (Figure 3), but no distinctive features or specific clusters were identified and their reported distension score $(4.7 \pm 0.2)$ was similar as in the rest $(4.6 \pm 0.1)$. Total gas volume measured in these patients with an abnormal gas pattern was $331[251,384] \mathrm{mL}$ and in 17 out of the 21 patients it was above the normal range (Figure 5). No consistent differences in intestinal gas volume and distribution were detected in relation to age, sex or BMI (data not shown). 


\section{DISCUSSION}

Our study, applying original analysis techniques on a relatively large cohort of subjects, provides unique and heretofore unavailable information on the volume and distribution of intestinal gas in normal conditions and in relation to functional gut symptoms.

We took advantage of the standardized acquisition procedure of CT imaging in our research program, that has been systematically applied over the past 10 years to pool a relatively large sample of patients with symptoms commonly attributed to excess gas production or accumulation in the gut lumen, such as bloating, abdominal distension and flatulence. This large cohort allowed the application of a sophisticated analysis approach by machine learning techniques. Provided with sufficient training data, the program learns to identify the combination of features, not necessarily abnormal if considered separately, that identify individuals outside the normal range.

During basal conditions, when patients were not experiencing symptoms, essentially no abnormalities were detected. Characteristically, bloating and abdominal distension often manifest as discrete, relatively brief and transient episodes. A key strategy in our program is the ability to acquire CT images on demand, and this approach allowed us to show that abnormal gas patterns develop in the symptomatic phase in a significant proportion of the same patients with normal gas features when asymptomatic. These observations further indicate that the content and distribution of gas in the human gut is very dynamic and that changes may keep a temporal relation to the occurrence of symptoms. Interestingly, the proportion of abnormalities detected during symptomatic episodes was similar to that observed in the subgroup of flatulent 
patients whose symptoms were exacerbated by a challenge diet, the only difference being a larger content of gas in the distal colon of the latter. A previous paired study in healthy subjects had shown a predominant accumulation of gas in the distal gut during the postprandial period (15), in analogy to the findings in patients on the challenge diet that were scanned after the test meal.

Conversely, it is intriguing that quite a large proportion of patients, even during symptomatic episodes, had showed an apparently normal pattern of intestinal gas. It has been consistently shown that patients with functional gut disorders complaining of episodic abdominal distension, even during basal conditions (i.e. when they are feeling well), manifest impaired transit and clearance of exogenous gas directly infused into the jejunum and develop symptoms in response to gas loads that are well tolerated by healthy subjects (4-9). Abnormalities in intestinal motor function have been also evidenced by endoluminal image analysis based on capsule endoscopy (22). This apparent discrepancy between prior data on abnormal motor function and normal gas content observed in part of our patient sample may be related to different factors. First, we acknowledge that in spite of the methodological refinements introduced in the present study, our imaging technique may remain insufficiently sensitive to detect some subtle abnormalities. Second, impaired gas transit evidenced by jejunal infusion studies concerns predominantly the small bowel, whereas gas is mostly produced in the colon. Third, the rate of gas infusion employed in the experimental studies was much higher than the normal production, since the objective was to evaluate the responses to a gas challenge. Furthermore, the gas mixture in previous studies mimicked the 
concentration of gases in venous blood to minimize absorption, and hence, contained a large proportion of nitrogen which, in contrast to the gases normally produced in the colon ( $\mathrm{CO}_{2}$ and hydrogen) is poorly absorbed and metabolized by colonic microbiota. Slow transit of endogenous gases would increase their absorption and consumption, and thereby reduce intraluminal volume $(1 ; 23)$.

In this paper, we present results that may be interpreted as consistent with the hypothesis that episodic and postprandial increases in intestinal gas are pathogenically related to certain functional gut symptoms. However, the relatively small magnitude of the changes and the fact that are only observed in part of the patient cohort, suggests that other key factors are involved. Indeed, it has been well documented that patients with functional gut symptoms manifest intestinal hypersensitivity that makes them more susceptible to distension and to other intraluminal stimuli (24-26). Specifically, patients complaining of abdominal bloating and distension have a poor tolerance of colonic gas loads administered transrectally compared to healthy subjects, and develop subjective symptoms as well as visible abdominal distension $(27 ; 28)$. The latter is a behavioral somatic response of the abdomino-thoracic muscles with a paradoxical contraction/descent of the diaphragm $(27 ; 28)$. Thus, we speculate that hypersensitive or hypervigilant patients may develop this type of aberrant responses to minor alterations or even normal gas content in the gut. Moreover, not only gas, but other components of intestinal content could contribute to the symptoms that patients may misinterpret as excess gas. The situation is distinctively different in patients with intestinal neuropathy and dysmotility evidenced by manometry, where the motor dysfunction is associated with 
massive pooling of intestinal gas and content that produces both abdominal distension and abdominal pain (12).

In conclusion, a sensitive and precise technology is now available to quantify intraluminal gas and its anatomic location. This information may be useful in a clinical setting where symptoms are related to excess gas in the gut by patients, attending physician or both. If little or no gas is demonstrated, evidence for distorted abdomino-thoracic muscular rearrangement as a cause for distension may be simultaneously obtained. 


\section{Acknowledgments}

The authors thank Anna Aparici, and Purificación Rodriguez for technical support and Gloria Santaliestra for secretarial assistance.

\section{Funding}

This work was supported in part by the Spanish Ministry of Economy and Competitiveness (Dirección General de Investigación Científica y Técnica, SAF 2013-43677-R); Ciberehd is funded by the Instituto de Salud Carlos III. Dr. Burri was supported by grants from the Freiwillige Akademische Gesellschaft (Basel, Switzerland) and the Gottfried und Julia Bangerter-Rhyner-Stiftung (Bern, Switzerland).

\section{Author Contributions:}

EBarba and $A B$ contributed equally as first authors.

AB. Data analysis

EBarba. Study management, conduction of experiments, and data analysis.

EBurri. Study management and conduction of experiments.

DC. Conduction of experiments.

$\mathrm{CM}$. Data analysis and manuscript revision.

SS. Data analysis.

AA. Study design, supervision of studies, data analysis and manuscript revision. $\mathrm{SQ}$. Analysis of CT images.

EM. Adaptation of CT analysis program.

IN. Adaptation of CT analysis program.

J-RM. Data interpretation, manuscript revision.

FA. Study design, data interpretation, and manuscript preparation.

\section{No competing interests declared}




\section{References}

(1) Azpiroz F. Intestinal gas. In: Feldman M, Friedman LS, Brandt LJ, editors. Sleisenger and Fordtran's. Gastrointestinal and Liver Disease: Pathophysiology, Diagnosis, Management. 10 ed. Canada: Saunders, Elsevier; 2013. p. in press.

(2) Azpiroz F, Malagelada J-R. Abdominal bloating. Gastroenterology 2005;129:1060-78.

(3) Agrawal A, Whorwell PJ. Review article: abdominal bloating and distension in functional gastrointestinal disorders--epidemiology and exploration of possible mechanisms. Aliment Pharmacol Ther 2008 Jan 1;27(1):2-10.

(4) Serra J, Salvioli B, Azpiroz F, Malagelada JR. Lipid-induced intestinal gas retention in the irritable bowel syndrome. Gastroenterology 2002;123:700-6.

(5) Passos MC, Tremolaterra F, Serra J, Azpiroz F, Malagelada J-R. Impaired reflex control of intestinal gas transit in patients with abdominal bloating. Gut 2005;54:344-8.

(6) Salvioli B, Serra J, Azpiroz F, Lorenzo C, Aguade S, Castell J, et al. Origin of gas retention and symptoms in patients with bloating. Gastroenterology 2005;128:574-9. 
(7) Serra J, Villoria A, Azpiroz F, Lobo B, Santos J, Accarino A, et al. Impaired intestinal gas propulsion in manometrically proven dysmotility and in irritable bowel syndrome. Neurogastroenterol Mot 2010;22:401-6.

(8) Serra J, Azpiroz F, Malagelada J-R. Impaired transit and tolerance of intestinal gas in the irritable bowel syndrome. Gut 2001;48:14-9.

(9) Caldarella MP, Serra J, Azpiroz F, Malagelada JR. Prokinetic effects in patients with intestinal gas retention. Gastroenterology 2002;122:174855.

(10) Accarino A, Perez F, Azpiroz F, Quiroga S, Malagelada J-R. Intestinal gas and bloating: effect of prokinetic stimulation. Am $\mathrm{J}$ Gastroenterol 2008;103:2036-42.

(11) Accarino A, Perez F, Azpiroz F, Quiroga S, Malagelada JR. Abdominal distension results from caudo-ventral redistribution of contents. Gastroenterology 2009;136:1544-51.

(12) Barba E, Quiroga S, Accarino A, Lahoya EM, Malagelada C, Burri E, et al. Mechanisms of abdominal distension in severe intestinal dysmotility: abdomino-thoracic response to gut retention. Neurogastroenterol Motil 2013 Apr 22;25(6):e-389-e394.

(13) Manichanh C, Eck A, Varela E, Roca J, Clemente JC, Gonzalez A, et al. Anal gas evacuation and colonic microbiota in patients with flatulence: effect of diet. Gut 2014;63:401-8. 
(14) Barba E, Burri E, Accarino A, Cisternas D, Quiroga S, Monclus E, et al. Abdomino-thoracic mechanisms of functional abdominal distension and correction by biofeedback. Gastroenterology 2014;in press.

(15) Perez F, Accarino A, Azpiroz F, Malagelada J-R. Gas distribution within the human gut: effect of meals. Am J Gastroenterol 2007;102:842-9.

(16) Ibañez L, Schroeder W, Ng L, Cates J. The ITK Software Guide. Kitware Inc 2003.

(17) Schroeder W, Martin K, Lorensen B. The visualization toolkit, an object oriented approach to 3D graphics. Kitware Inc 2002;3rd edition.

(18) Camci F, Chinnam RB. General support vector representation machine for one-class classification of non-stationary classes. 2008 Oct;41(10):3021-3034. Pattern Recognition 2014;41(10):3021-34.

(19) Lloyd SP. Least-Squares Quantization in Pcm. leee Transactions on Information Theory 1982;28(2):129-37.

(20) Stone M. Cross-validatory choice and assessment of statistical predictions (with discussion). J Roy Stat Soc B 1974;36:111-47.

(21) Thompson WG, Longstreth G, Drossman DA, Heaton K, Irvine EJ, Muller-Lissner S. Functional bowel disorders and Functional abdominal pain. In: Drossman DA, Corazziari E, Talley NJ, Thompson WG, Whitehead WE, editors. The Functional Gastrointestinal Disorders. 2nd ed. Mc Lean, VA: Degnon Associates; 2000. p. 351-432. 
(22) Malagelada C, De lorio F, Segui S, Mendez S, Drozdzal M, Vitria J, et al. Functional gut disorders or disordered gut function? small bowel dysmotility evidenced by an original technique. Neurogastroenterol Mot 2012;24:223-e-105.

(23) Mego M, Bendezú A, Accarino A, Malagelada J-R, Azpiroz F. Intestinal gas homeostasis: disposal pathways. Neurogastroenterol Motil 2014;in press.

(24) Azpiroz F. From sensation to perception: the gut brain connection. In: Pasricha J, Willis WD, Gebhart GF, editors. Chronic Abdominal and Visceral Pain. Theory and Practice.Boca Raton FL: CRC Press; 2007. p. 193-203.

(25) Kellow JE, Azpiroz F, Delvaux M, Gebhart GF, Mertz H, Quigley EM, et al. Applied principles of neurogastroenterology: physiology/motility sensation. Gastroenterology 2006;130:1412-20.

(26) Azpiroz F, Bouin M, Camilleri M, Mayer EA, Poitras P, Serra J, et al. Mechanisms of hypersensitivity in IBS and functional disorders. Neurogastroenterol Mot 2007;19:62-88.

(27) Villoria A, Azpiroz F, Burri E, Cisternas D, Soldevilla A, Malagelada J-R. Abdomino-phrenic dyssynergia in patients with abdominal bloating and distension. Am J Gastroenterol 2011;106:815-9.

(28) Tremolaterra F, Villoria A, Azpiroz F, Serra J, Aguade S, Malagelada JR. Impaired viscerosomatic reflexes and abdominal wall dystony associated with bloating. Gastroenterology 2006;130:1062-8. 


\section{FIGURE LEGENDS}

Figure 1. Total abdominal and colonic gas content. Data are shown as boxplots in healthy subjects and in patients with various symptomatic states.

Figure 2. Gas content in different gut compartments. Data are shown as boxplots in healthy subjects and in patients with various symptomatic states.

Figure 3. Proportion of subjects identified by the classifier within and without the normal range (upper panel) and their number of abnormal features (lower panel).

Figure 4 Gas in the small bowel: distribution by bubble size. Bubbles are pooled by their size in three groups. Data are shown as boxplots in healthy subjects and in patients with various symptomatic states.

Figure 5 Examples of abdominal gas. Three-dimensional reconstruction from CT scans in a healthy subject $(70 \mathrm{~mL}$ total volume, $47 \mathrm{~mL}$ in colon) and a patient outside the normal range $(383 \mathrm{~mL}$ total volume, $363 \mathrm{~mL}$ in colon). 